\title{
Drugs- $\beta$-Cyclodextrin inclusion complex: Would be a new strategy to improve Antihypertensive Therapy?
}

\author{
Quiara Lovatti Alves, Samuel Barbosa Camargo, Rafael Leonne Cruz de Jesus and Darízy Flávia Silva* \\ Laboratory of Cardiovascular Physiology and Pharmacology, Federal University of Bahia, Salvador, Bahia, Brazil
}

\begin{abstract}
Taking into account the increasing use of cyclodextrins (CDs) to overcome drawbacks of drugs like antihypertensive, complex formation with of CDs seems to be a good pharmaceutical strategy to improve stability, solubility, bioavailability and reduction of side effects, thus facilitating the control of hypertension. The main objective of this short communication is to demonstrate the antihypertensive potential of complexed compounds with $\beta$-cyclodextrins and their clinical applications. This short communication identified and illustrated that $\beta$-cyclodextrin-based systems are promising sources that provide improved pharmacokinetic and formulation conditions, and important applications have been established by many research groups, optimizing $\beta$-CDs derivative systems for oral drug therapy, especially in hypertension.
\end{abstract}

\section{Introduction}

Cyclodextrins (CDs) are a family of cyclic oligosaccharides that have been largely studied and used industrially in pharmaceutical applications, due the fact they are able to form inclusion complexes with poorly water-soluble drugs and improve a variety of drug properties, such as stability, dissolution rate, solubility, bioavailability and reduction of side effects [1-3]. CDs are composed of at least $6 \mathrm{D}-(+)$ glucopyranose units attached by $\alpha-(1,4)$ glucosidic bonds that differ in their ring size and solubility [4]. There are 3 important subtypes that naturally occur: $\alpha-C D, \beta-C D$, and $\gamma$-CD with 6,7 and 8 glucopyranoside units respectively [4]. They became scientifically attractive due to their application potential and important impact in hypertensive therapy. The most antihypertensive drugs are orally administered [5] and according the Biopharmaceutical Classification System (BCS) there are two important parameters that control rate and extent of drug absorption. These include drug dissolution and gastrointestinal permeability, which are considered the variables that most affecting drug bioavailability [6]. Many antihypertensive drugs are classified by BCS, in different classes, including: low solubility and high permeability characteristics after oral administration (class II), high solubility and low permeability (class III) and low solubility and permeability (class IV) [6-8]. Furthermore, controlled-release formulations of some antihypertensive drugs are unstable both in vivo and in vitro when administered orally [9], thus resulting in a lack of suitable specific antihypertensive formulations, especially for pediatric patients [10]. Taking this into account, CDs have been used to circumvent these limitations, and forming complexes with antihypertensives and CDs is a promising pharmaceutical strategy to facilitate the management of hypertension. Thus, the main objective of this short communication is to demonstrate the antihypertensive potential of compounds complexed with $\beta$-cyclodextrins and their clinical applications.

\section{Applications of $\beta-C D$ in Hypertension Treatment}

For many drugs, the cavity size of $\alpha$-CDs is unsatisfactory while $\gamma$-CDs is more expensive. The $\beta$-CDs has been extensively used for its immediate availability and cavity size suitable for a wide range of medications. Despite this, the use of $\beta$-CDs is limited due to low solubility in water and nephrotoxicity, especially in parenteral preparations [11]. However, when administered orally, cyclodextrins are considered safe and void of toxicity, as well as low bioavailable [12] and therefore is not sufficiently absorbed into the gastrointestinal system [13]. After the discovery of $\beta$-CD's promising properties, it was possible to study new formulations for antihypertensive drugs. Although few studies have assessed the inclusion complexes of drugs with $\beta-C D$ in cardiovascular diseases, our group demonstrated that linalool, a vasodilator [14] and hypotensive monoterpene [15], complexed with $\beta$-CD promoted a significant decrease in the mean arterial pressure of spontaneously hypertensive rats (SHR) when compared to the non-complexed form, an effect that may be related to the increased stability and bioavailability afforded by $\beta$-CD [16]. Another monoterpene, $\beta$-Pinene, when complexed with $\beta-C D$ significantly reduced mean arterial pressure when compared to free $\beta$-Pinene in a model of hypertension induced by L-NAME. Thus, $\beta$-Pinene complex formation with $\beta$-CD improved the pharmacological profile of this monoterpene [17].

An important derivative of $\beta$-CD is methyl- $\beta$-cyclodextrin (M- $\beta$ $\mathrm{CD}$ ), a cholesterol binding agent. Cardiovascular studies performed in apolipoprotein $\mathrm{E}(\mathrm{ApoE} 2 / 2)$ deficient mice, where infusing angiotensin II (Ang II) promotes atherosclerosis and abdominal aortic aneurysm, and in this model, an increase in blood pressure has been reported. Isolated aortas from Ang II-infused mice were significantly less responsive to acetylcholine-induced endothelium-dependent relaxation when compared to aortas infused with vehicle control. Preincubation with $\mathrm{M}-\beta-\mathrm{CD}$ in isolated aortic rings from this animals,

*Correspondence to: Darízy Flávia Silva, $\mathrm{PhD}$, Laboratory of Cardiovascular Physiology and Pharmacology, Federal University of Bahia, Avenida Reitor Miguel Calmon, Vale do Canela, 40110-902 Salvador, Bahia, Brazil, E-mail: darizy@gmail.com

Received: July 09, 2019; Accepted: August 01, 2019; Published: August 05, 2019 
resulted in an acetylcholine-induced relaxation that was significantly greater than when compared with vehicle pre-incubation, suggesting that it partially restored the acetylcholine-induced aortic relaxation in the angiotensin II-infused mice [18]. Despite this, it has already been reported that $\beta-C D$ and $M-\beta-C D$, but not $\alpha-C D, \gamma-C D$, and HP- $\beta$ $\mathrm{CD}$, are capable of inducing caspase-dependent apoptotic cell death in human keratinocytes on depletion of membrane cholesterol [19]. Therefore, more studies are needed to ensure their safety and toxicity in other cell types.

The cardiovascular effect of the complex hydroxypropyl- $\beta$ cyclodextrin (HP- $\beta-\mathrm{CD}$ ), another derivative of $\beta-\mathrm{CD}$, and Angiotensin (1-7) [(Ang-(1-7)], a cardioprotective metabolite of Angiotensine I and Angiotensine II ([20] was evaluated. The assays were performed with four experimental groups: sedentary treated with tap water, sedentary treated with oral administration of HP- $\beta$-CD/Ang-(1-7) inclusion compound, exercise-trained treated with tap water and exercise-trained treated with HP- $\beta-\mathrm{CD} /$ Ang-(1-7). Treatment was administered by gavage over the course of 10 days, concomitantly with the exercise training protocol. The complex resulted in a reduction of blood pressure, improved cardiovascular autonomic control, attenuated cardiac hypertrophy and restored left ventricular function. These results were similar to those produced by physical training in SHR, while the combined effect of both interventions potentiate the impact on blood pressure. It is important to note that the inclusion compound had no adverse effects on SHR. Additionally, chronic administration of beneficial cardiovascular responses of the HP- $\beta-\mathrm{CD} /$ Ang-(1-7) inclusion compound obtained with Ang-(1- 7) orally active also include improvement of left ventricular function and mechanical properties of cardiac muscle [21].

A relevant example of $\beta-C D$ application can be found in pediatric formulations. As there is a lack of specific formulations which are suitable for pediatric treatment, individuals tend to disrupt or open capsules by breaking and/or crushing tablets, and even dilute liquid dosage in order to achieve the prescribed dosage. This effort to achieve a more easily administrable preparations for this group of patients, causes reduced precision in respect to dose and bioavailability, as well as the chemical, physical and microbiological stability of the formulation $[22,23]$. In children, administering in the liquid formulation tends to be preferred, due to their wider acceptability and dose flexibility [24]. The hydrochlorothiazide (HTZ) is one of the most commonly used diuretics in the treatment of hypertension, however commercial liquid formulations of HTZ are not available due to its limited solubility [25] and low stability in aqueous solution [26], leading to complications in the administration of HTZ in pediatric patients. Regardless, HTZ is the only FDA-approved diuretic for children [27]. To address this challenge, an innovative pediatric oral formulation of HTZ $(2 \mathrm{mg} /$ $\mathrm{mL}$ ) was developed by combining the drug- $\beta-\mathrm{CD}$ complexation with the incorporation of the complex into solid lipid nanoparticles, which demonstrated an increase in both the diuretic effect and the sustained drug release that led to an increased oral bioavailability of HTZ after complex formation [10].

Another drug commonly used for pediatric hypertension treatment is ramipril [28]. Ramipril is a highly lipophilic and poorly water-soluble drug with very low bioavailability when compared to the intravenous administration [29,30]. To prepare an oral formulation, ramipril was solubilized through complex formation with hydroxypropyl$\beta$-cyclodextrin (HP- $\beta$-CD), a modified $\beta$-CD that offers improved solubility when compared to the $\beta-C D$. This systematic optimization of formulation parameters resulted in the development of oral liquid ramipril, a product that is stable for 12 months, offering preferential pediatric use over existing alternatives [31]. However, one of the most common side effects of ramipril use is diarrhea [32] and cyclodextrin are capable of stimulating intestinal secretion and gastrointestinal propulsion in animals, causing diarrhea. The increased gastrointestinal motility may be a result of the complex formation of bile salts with cyclodextrin, which leads to increased intestinal lipid concentrations [33]. Thus, it is possible that the combination of ramipril and CDs can intensify this adverse effect, leading to increased patients suffering from these undesirable effects. There is a dearth of clinical trial data regarding the safety and efficacy of antihypertensive drugs used in children, and many FDA-approved adult drugs are not effective in pediatric hypertension studies, thus dosage, safety and efficacy of the pediatric drug cannot be extrapolated from adult clinical trials [34].

\section{Final Considerations}

The $\beta$-cyclodextrin-based systems are currently being studied and are promising sources aimed at providing improved conditions for pharmacokinetic and formulation design efficiency. This short communication identified and illustrated important applications which have been studied by several research groups that formulate and optimize $\beta$-CDs derivates systems for oral drug therapy in hypertension.

\section{References}

1. Davis ME, Brewster ME (2004) Cyclodextrin-based pharmaceutics: Past, present and future. Nat Rev Drug Discov 3: 1023-1035.

2. Loftsson T, Duchêne D (2007) Cyclodextrins and their pharmaceutical applications. Int J Pharm 329: 1-11.

3. Scalia S, Villani S, Casolari A (1999) Inclusion complexation of the sunscreen agent 2-ethylhexyl-p-dimethylaminobenzoate with hydroxypropyl-beta-cyclodextrin: effect on photostability. JPP 51: 1367-1374.

4. Rajeswari C, Alka A, Javed A, Kha R (2005) Cyclodextrins in drug delivery: an updated review. AAPS PharmSciTech 6: 329-357.

5. Nguyen Q, Dominguez J, Nguyen L, Gullapalli N (2010) Hypertension management: An update. Am Health Drug Benefits 3: 47-55.

6. Amidon GL, Lennernäs H, Shah VP, Crison JR (1995) A theoretical basis for a biopharmaceutic drug classification: the correlation of in vitro drug product dissolution and in vivo bioavailability. Pharm Res 12: 413-420.

7. Löbenberg R, Amidon GL (2000) Modern bioavailability, bioequivalence and biopharmaceutics classification system. New scientific approaches to international regulatory standards. European Journal of Pharmaceutics and Biopharmaceutics 50: 3-12.

8. Mendes C, Buttchevitz A, Kruger JH, Kratz JM, Simões CMO, et al. (2016) Inclusion complexes of hydrochlorothiazide and B-cyclodextrin: Physicochemical characteristics, in vitro and in vivo studies. Eur J Pharm Sci 83: 71-78.

9. de Azevedo M de BM, Tasic L, Fattori J, Rodrigues FHS, Cantos FC, et al. (2011) New formulation of an old drug in hypertension treatment: the sustained release of captopril from cyclodextrin nanoparticles. Int J Nanomedicine 6: 1005-1016.

10. Cirri M, Mennini N, Maestrelli F, Mura P, Ghelardini C, et al. (2017) Development and in vivo evaluation of an innovative "Hydrochlorothiazide-in Cyclodextrinsin Solid Lipid Nanoparticles" formulation with sustained release and enhanced oral bioavailability for potential hypertension treatment in pediatrics. International Journal of Pharmaceutics 521: 73-83.

11. Szejtli J (1991) Cylodextrin in drug formulations: Part I. Pharm Technol Int 5: 556-567.

12. Brewster ME, Loftsson T (2007) Cyclodextrins as pharmaceutical solubilizers. $A d v$ Drug Deliv Rev 59: 645-666.

13. Irie T, Uekama K (1997) Pharmaceutical applications of cyclodextrins. III Toxicological issues and safety evaluation. J Pharm Sci 86: 147-162.

14. Rice GI, Thomas DA, Grant PJ, Turner AJ, Hooper NM (2004) Evaluation of angiotensin-converting enzyme (ACE), its homologue ACE2 and neprilysin in angiotensin peptide metabolism. Biochem J 383(Pt 1): 45-51. 
15. Menezes IAC, Barreto CMN, Antoniolli ÂR, Santos MRV, de Sousa DP (2010) Hypotensive activity of terpenes found in essential oils. Journal of Biosciences 65 : 562-566.

16. Camargo SB, Simões LO, Medeiros CF de A, de Melo Jesus A, Fregoneze JB, et al. (2018) Antihypertensive potential of linalool and linalool complexed with B-cyclodextrin: Effects of subchronic treatment on blood pressure and vascular reactivity. Biochem Pharmacol 151: 38-46.

17. Moreira IJA, Menezes PP, Serafini MR, Araújo AAS, Quintans-Júnior LJ, et al. (2016) Characterization and Antihypertensive Effect of the Complex of (-)- $\beta$ - pinene in B-cyclodextrin. Curr Pharm Biotechnol 17: 837-845.

18. Seto SW, Krishna SM, Yu H, Liu D, Khosla S, et al. (2013) Impaired AcetylcholineInduced Endothelium-Dependent Aortic Relaxation by Caveolin-1 in Angiotensin IIInfused Apolipoprotein-E (ApoE -/- ) Knockout Mice. PLOS ONE p. 8.

19. Schönfelder U, Radestock A, Elsner P, Hipler UC (2006) Cyclodextrin-induced apoptosis in human keratinocytes is caspase- 8 dependent and accompanied by mitochondrial cytochrome c release. Experimental Dermatology 15: 883-890.

20. Santos RAS, Ferreira AJ, Simões E Silva AC (2008) Recent advances in the angiotensinconverting enzyme 2-angiotensin(1-7)-Mas axis. Exp Physiol 93: 519-527.

21. Bertagnolli M, Casali KR, De Sousa FB, Rigatto K, Becker L, et al. (2014) An orally active angiotensin-(1-7) inclusion compound and exercise training produce similar cardiovascular effects in spontaneously hypertensive rats. Peptides 51: 65-73.

22. Brion F, Nunn AJ, Rieutord A (2003) Extemporaneous (magistral) preparation of oral medicines for children in European hospitals. Acta Paediatrica 92: 486-490.

23. Standing JF, Tuleu C (2005) Paediatric formulations - Getting to the heart of the problem. Int J Pharm 300: 56-66.

24. Kristensen HG (2012) WHO guideline development of paediatric medicines: points to consider in pharmaceutical development. Int J Pharm 435: 134-135.
25. Basanta Kumar Reddy B, Karunakar A (2011) Biopharmaceutics classification system: A regulatory approach. Dissolution Technologies 18: 31-37.

26. Mollica JA, Rehm CR, Smith JB (1969) Hydrolysis of hydrochlorothiazide. Journal of Pharmaceutical Sciences 58: 635-636.

27. National High Blood Pressure Education Program Working Group on High Blood Pressure in Children and Adolescents (2004) The fourth report on the diagnosis, evaluation, and treatment of high blood pressure in children and adolescents. Pediatrics 114(2 Suppl 4th Report): 555-576.

28. Paediatric Formulary Committee (2011) Paediatric Formulary Committee. BNF for children 2011-2012 (British National Formulary for Children). Pharmaceutical Press, London.

29. Levitt DG, Schoemaker RC (2006) Human physiologically based pharmacokinetic model for ACE inhibitors: Ramipril and ramiprilat. BMC Clin Pharmacol 6: 1-27.

30. van Griensven JMT, Schoemaker RC, Cohen AF, Luus HG, Seibert-Grafe M, et al. (1995) Pharmacokinetics, pharmacodynamics and bioavailability of the ACE inhibitor ramipril. European Journal of Clinical Pharmacology 47: 513-518.

31. Russell C, Begum S, Hussain Y, Hussain M, Huen D, et al. (2015) Paediatric drug development of ramipril: reformulation, in vitro and in vivo evaluation. $J$ Drug Target 23: 854-863. [Crossref]

32. Carré A, Vasmant D, Elmalem J, Thiéry $\mathrm{P}$ (1991) Tolerability of ramipril in a multicenter study of mild-to-moderate hypertension in general practice. J Cardiovasc Pharmacol 18 Suppl 2: S141-S143.

33. Stevens DA (1999) Itraconazole in cyclodextrin solution. Pharmacotherapy 19: 603611. [Crossref]

34. Chu PY, Campbell MJ, Miller SG, Hill KD (2014) Anti-hypertensive drugs in children and adolescents. World J Cardiol 6: 234-244. [Crossref]

Copyright: (C2019 Alves QL. This is an open-access article distributed under the terms of the Creative Commons Attribution License, which permits unrestricted use, distribution, and reproduction in any medium, provided the original author and source are credited. 